\title{
Small Lymphocytic Lymphoma
}

National Cancer Institute

\section{Source}

National Cancer Institute. Small Lymphocytic Lymphoma. NCI Thesaurus. Code C7540.

A non-Hodgkin lymphoma composed of monomorphic small, round B-lymphocytes in the lymph nodes. When the lymphoid process predominantly involves the bone marrow and the peripheral blood it is called chronic lymphocytic leukemia. (WHO, 2001) 